\title{
Hospital Length of Stay and Readmission Rate for Neurosurgical Patients
}

Shaheryar F Ansari, MD ${ }^{1}$, Hong Yan, BS ${ }^{2}$, Jian Zou, $\mathrm{PhD}^{2}$, Robert M. Worth, $\mathrm{MD}^{1,2,3}$,

Nicholas M. Barbaro, $\mathrm{MD}^{1,3}$

${ }^{1}$ Indiana University Department of Neurological Surgery, Indianapolis, Indiana;

${ }^{2}$ Worcester Polytechnic Institute Department of Mathematical Sciences, Worchester, Massachusetts; ${ }^{3}$ Goodman Campbell Brain and Spine, Indianapolis, Indiana

\section{Email addresses:}

Shaheryar F Ansari: sfansari@iu.edu

Hong Yan: hyan@wpi.edu

Jian Zou: jzou@wpi.edu

Robert M. Worth: rworth@iupui.edu

Nicholas M. Barbaro: nbarbaro@goodmancampbell.com

\section{Corresponding author:}

Nicholas M. Barbaro MD

Goodman Campbell Brain and Spine

Indiana University Department of Neurosurgery

355 W. 16th St. Suite 5100

Indianapolis, IN 46202

Phone: 317-396-1300

Fax: 317-924-8472

nbarbaro@goodmancampbell.com

Disclosure: This paper has not been previously presented or published, in whole, or in part, and is not under consideration by any other publisher. The authors have no personal or financial conflicts of interest. The authors received no funding for this research.

This is the author's manuscript of the article published in final edited form as:

Ansari, S. F., Yan, H., Zou, J., Worth, R. M., \& Barbaro, N. M. (2018). Hospital Length of Stay and Readmission Rate for Neurosurgical Patients. Neurosurgery, 82(2), 173-181. https://doi.org/10.1093/neuros/nyx160 


\begin{abstract}
Background: Hospital readmission rate has become a major indicator of quality of care, with penalties given to hospitals with high rates of readmission. At the same time, insurers are increasing pressure for greater efficiency and reduced costs, including decreasing hospital lengths of stay (LOS).

Objective: The authors analyze their own service to determine if there is a relationship between LOS and readmission rates.
\end{abstract}

Methods: Records of patients admitted to the authors' institution from October 2007 through June 2014 were analyzed for several data points, including initial LOS, readmission occurrence, admitting and secondary diagnoses, and discharge disposition.

Results: Out of 9,409 patient encounters, there were 925 readmissions. Average LOS was 6 days. Univariate analysis indicated a higher readmission rate with more diagnoses upon admission $(\mathrm{p}<0.001)$ and an association between insurance type and readmission $(\mathrm{p}<0.001)$, as well as decreasing average yearly $\operatorname{LOS}(\mathrm{p}=0.0045)$. Multivariate analysis indicated statistically significant associations between longer LOS $(p=0.03)$ and government insurance $(\mathrm{p}<0.01)$.

Conclusion: A decreasing LOS over time has been associated with an increasing readmission rate at the population level. However, at the individual level, a prolonged LOS was associated with a higher risk of readmission. This was attributed to patient comorbidities. However, this increasing readmission rate may represent many factors including patients' overall health status. Thus the rate of readmission may represent a burden of illness rather than a valid metric for quality of care.

Key words: hospital costs; patient readmissions; quality indicators, health care

Short title: Hospital Length of Stay and Readmission Rate 


\section{INTRODUCTION}

Over the past several years, government and private insurers have made efforts to decrease the number of days patients spend in the hospital as a way to drive down healthcare costs. At the same time, there have arisen penalties for hospitals for patients being readmitted to the hospital within 30 days of discharge, ${ }^{1,2}$ based on a report by the Centers for Medicare and Medicaid Services (CMS) on the purported costs to the government of early readmissions. ${ }^{3}$ These reports suggest that early readmissions are a substantial cost to the overall healthcare system - in 2003-2004, almost one-fifth of Medicare beneficiaries were readmitted within 30 days of hospital discharge, incurring an estimated cost of $\$ 17$ billion. ${ }^{1}$ Much of this cost was incurred by patients with chronic diseases such as congestive heart failure and chronic obstructive pulmonary disease; both diseases present a significant public health burden. ${ }^{4}$ For this reason, the CMS has focused first on these conditions, along with myocardial infarction and pneumonia. Much research has been performed and published on these medical conditions and prevention of early readmission after hospitalization. ${ }^{1,2,5-8}$ In 2015 , elective total hip and knee arthroplasties were also included in the group of monitored conditions. ${ }^{9}$ As a result of the substantial costs incurred due to early readmissions, the CMS has begun to use this measure as an indicator of hospital and provider quality, seemingly associating a lower readmission rate with a higher quality of care, and vice versa.

In the neurosurgical literature, there is a growing body of publications regarding the factors associated with readmission rates. ${ }^{10-14}$ Many conditions treated by neurosurgeons are devastating, and some patients may require a prolonged period of intensive care and monitoring before they are well enough to be discharged. Furthermore, many neurosurgical patients continue to require care after hospital discharge and occasionally need to return to the hospital if their condition declines. Patients often return if they suffer a decline or their needs exceed the capabilities of the facility to which they were discharged. As a result, neurosurgical patients may have higher readmission rates than patients with similar overall health status and non-neurosurgical conditions. ${ }^{13}$ In our institution, as in many others, there is a desire to reduce the costs and improve the quality of care. One way to reduce costs is to decrease the length of hospital stay (LOS), and this 
has resulted in more outpatient procedures and many patients being discharged on the first day after surgery. However, these two pressures - to reduce LOS while also minimizing readmissions - are potentially at odds with each other. No previous study has compared LOS with readmission in the neurosurgical population. We hypothesized that reducing LOS would result in an increased readmission rate. To evaluate this, the hospital administrative database was queried for all patients admitted to the neurosurgery service from October 2007 through June 2014, and this cohort was analyzed for a relationship between hospital LOS, along with other factors, and readmission rate.

\section{METHODS}

\section{Study Design}

Patient records were retrospectively obtained from a hospital administrative database for all adult patients admitted to the neurosurgical service at Indiana University Health Methodist Hospital from October 2007 through June 2014. The record review was performed in September 2014 in order to include patients from the last month of the series. The patient selection is diagrammed in Figure 1. October 2007 was chosen as the beginning of the study period because this was when the institution began using electronic medical records (EMR) and the data collected and stored such that it could be used in the study. Readmission was defined as admission to the hospital on any service within 30 days of prior discharge. Length of stay was defined according to the hospital administrative database, which defines a hospital day as a day in which the patient occupied a bed at midnight. The inclusion criteria were as follows: adult patients admitted to the neurosurgical service either electively, through the emergency department, or directly from clinic. There were 9,739 patient encounters during this time. The following data points were collected for each patient: demographics, dates of admission and discharge, admission priority, discharge disposition, admission diagnoses, and procedure(s) performed. The records were anonymized and analyzed for LOS, whether or not patients were readmitted, correlation between LOS and readmission, correlation between discharge disposition and readmission, correlation between admission priority and readmission, and correlation between type of surgery (cranial vs spinal) and readmission. Admission priority was classified as either elective or non- 
elective (emergency, direct admission). Discharge disposition was classified as home, rehab (acute and subacute), other. The authors also analyzed reasons for readmission. Patients who died were excluded from the analysis.

\section{Statistical Methods}

In determining the likelihood of readmission relative to categorical variables, a Cox proportional hazard model was employed. A two-sample unpaired student's $t$ test was used when two sets of numbers needed to be compared for similarity. Fisher's exact test was used to compare two cohorts when needed. All statistical analysis was carried out in SAS 9.4.

\section{RESULTS}

\section{Patient Demographics}

There were 9,739 patient encounters on the adult neurosurgical service from October 2007 through June 2014. A total of 330 of these patients died and were therefore not included in the analysis, leaving 9,409 encounters. There were 925 early readmissions during this time (9.8\%). Table 1 shows the demographics of this group. Approximately half of the population was male, and 5,916 or $62.9 \%$ of the admissions were elective. The average $\operatorname{LOS} \pm$ standard deviation (SD) for all initial admissions was $6.21 \pm 7.4$ days. Table 2 details the classifications of patients who were readmitted - in an elective fashion (ie, from clinic or as a direct admission from an outside facility) vs through the emergency department. Patients with a neurological reason for readmission included those with complications from neurosurgical operations, meningitis, postoperative pain, postoperative seizures, and hydrocephalus, and comprised $36.6 \%$ of the readmitted cohort. Detailed breakdown of this group is shown in Table 3. Patients with infections in the surgical site (non-meningitis) were considered as a separate group; they were grouped differently in the hospital database and they comprised $8.0 \%$ of the group. Patients with ventriculoperitoneal shunt malfunctions were classified as "device-related," whereas patients with shunt infections were included in the "postop infection" grouping.

Patients with a "medical" reason for readmission included problems with other body systems, were 362 in number and comprised $39.1 \%$ of readmissions. Table 4 
provides an overview of the medical readmissions based on coding in the database utilized for the study. Gastrointestinal (GI) diagnoses, which consisted of gastroesophageal reflux, severe nausea/vomiting, constipation/diarrhea, and GI bleeding, among others, accounted for the largest group of readmitted patients with 68 (18.8\%). The next largest group were those with hematologic disorders, including DVT/PE or bleeding disorders, 63 (17.4\%). Patients with complications related to systemic malignancy, cardiac disease, and renal/urinary disorders (including UTIs) were 50 $(13.8 \%), 49(13.5 \%)$, and $49(13.5 \%)$ in number, respectively. There were 38 patients $(10.5 \%)$ with pulmonary disease, primarily relating to COPD, and 30 patients with medication-related problems $(8.3 \%)$. Finally, patients with endocrine disorders, including diabetes mellitus and disorders of the pituitary axis were 15 in number $(4.1 \%)$ The “other" category contained 59 patients and included unrelated musculoskeletal pain resulting in readmission, as well as psychiatric admissions and nonsurgical wound problems (eg, in trauma patients with multiple injuries), and this category included $6.4 \%$ of the group.

The authors analyzed readmission rate with regard to insurance status. The classes of insurance listed in the administrative database included Commercial, Medicare, Medicaid, Self Pay, Other government, and Workman's Comp. These were distilled into Commercial, Government, Self Pay, and Workman's Comp. The distribution of the population is detailed in Table 5. Of 3959 patients with commercial insurance, 322 $(8.1 \%)$ were readmitted; 544 of 4849 patients with government insurance $(11.2 \%)$ were readmitted. 66 of 530 self-pay patients were readmitted (10.6\%) and 3 of 71 Workman's Comp patients $(4.2 \%)$ were readmitted. Patients with government insurance were significantly more likely to be readmitted than patients with private insurance $(\mathrm{p}<0.0001)$, who were also significantly less likely to be readmitted than self-pay patients $(\mathrm{p}=0.04)$.

Univariate analysis of readmission with regard to number of diagnoses indicated that readmission was significantly associated with comorbidity. Table 6 shows that among 4909 patients with less than 10 diagnoses, 413 were readmitted (8.53\%), whereas 512 of 4500 patients with more than 10 present-on-admission diagnoses $(11.38 \%)$ were 
readmitted. These groups were significantly different $(\mathrm{p}<0.001)$. Univariate analysis of LOS, as outlined in Table 7, indicates that patients with more than 10 diagnoses had a significantly longer LOS than patients with a less than 10 diagnoses on admission $(p<0.01)$. Average yearly LOS was found to have a strong negative linear correlation with readmission rate (Figure $2, \mathrm{p}=0.0045$ ). Thus on a population level, the decreasing LOS is associated with an increasing readmission rate. It should be noted that for year 2007 , the rate of readmission was $6 \%$ and included only data from October through December of that year, a figure which the authors believe to be representative of the whole year. It should be noted that the switch to an EMR system occurred at this time, allowing this data to be collected.

The categorical variables of encounter type (initial vs readmission), gender, race, admission priority, and discharge disposition were subjected to the Cox proportional hazard analysis. The two continuous variables in the model, age and LOS, were dichotomized to make them categorical variables. Age was dichotomized as under 65 or over 65. LOS was discretized into quartiles, thus converting it into a categorical variable, and risk-adjusted for MS-DRG. Number of diagnoses, indicative of comorbidities, was dichotomized as less than 10 vs greater than 10 diagnoses present on admission. Table 8 shows the results of the Cox proportional multivariate analysis, indicating the hazard ratio (HR) for readmission to the hospital within 30 days and the $95 \%$ confidence interval for the HR

The multivariate analysis indicates that patients within the upper quartile of adjusted LOS have a higher risk of readmission than patients in the lowest quartile $(\mathrm{p}=0.03)$. Thus individual patients who have a longer LOS have a higher likelihood of being readmitted. Gender, age, race, admission priority, number of diagnoses, discharge disposition, and MS-DRG were not found to be significantly associated with readmission rate (though age did approach statistical significance). Government insurance did have a significantly higher association with readmission than commercial insurance. Number of diagnoses, representative of comorbidity, seemed to lose significance in the multivariate analysis, though it showed strong statistical significance in univariate analysis. 


\section{DISCUSSION}

\section{Key Findings}

The authors performed an analysis of admissions to their service at one hospital to determine factors associated with early hospital readmission. The overall readmission rate of $9.8 \%$ was similar to rates cited in the literature for readmission to neurosurgical services. ${ }^{11-13,15,16}$ The Vizient ${ }^{\mathrm{TM}}$ database was queried for LOS and readmission information among comparable-size institutions to our own (1000-1600 reported cases/year). ${ }^{17}$ This comparison is diagrammed in Figure 3. Our institution falls largely in the middle of the group with regard to LOS and readmission and does not represent an outlier among similar-sized institutions. It should be noted that the data from Vizient only covered Quarter 4 of 2012 through Quarter 2 of 2014, as this was the only time period available at the time of query. More patients were readmitted for medical reasons than for any other reason in our study. Surgical site infections and hardware complications made up a relatively small proportion of readmissions in this study. These findings are corroborated by others who have previously published on this topic. ${ }^{12}$ In the current health insurance and delivery climate, readmission has come to be equated with poor performance of providers and hospitals and is now tied to reimbursement for both. ${ }^{2,5,13}$ At the same time, pressure is added to reduce the length of time spent in the hospital. $A$ priori, it would seem that the goals of shorter LOS and decreased readmission rate are negatively correlated and that earlier discharges might paradoxically drive patients back to the hospital when they suffer a complication. ${ }^{7}$ Clarification of this relationship was the motivation for the current study.

Reasons for readmission in this study were classified as neurological problems, medical complications, and other problems unrelated to the patients' neurological or medical issues. Medical problems were the most common reason for readmission, with gastrointestinal issues accounting for the greatest proportion of readmission diagnoses, followed by oncologic complications. Among the neurological reasons for readmission, the most common were postoperative hematoma/seroma and cerebrospinal fluid leakage that required a return to the operating room. The nature of the hospital database did not allow a more in-depth analysis of this area, however, it seems that our study differs from 
the results of some others in that systemic comorbidities resulted in more readmissions than surgical complications requiring reoperation. ${ }^{12-14,18}$

The univariate analysis of the effect of insurance status on readmission indicated that patients with government insurance or those who were uninsured were more likely to be readmitted than patients with commercial insurance or those whose claims were handled through Workman's Compensation. One of the reasons is immediately evident patients with no insurance or limited Medicaid services may not have access to a primary care provider who can address problems in an office setting or over the phone. Furthermore, patients with Medicare are more likely to be older and have medical comorbidities which might predispose them to readmission. This finding is in line with that of Lavernia, et al, who found that patients with government or no insurance were more likely to be readmitted following hip arthroplasties than patients with commercial insurance. ${ }^{19}$ This study examined a larger cohort than is studied in the present analysis (over 27,000 patients) and had a significantly larger Medicare population (71\%), though our study has a larger proportion of Medicaid and self-pay patients. The important conclusion of the Lavernia paper, which is supported by our work here, is that readmission should not be viewed as a monodimensional measure of quality of a hospital, but rather is a multifactorial element, of which socioeconomic status is one contributor. ${ }^{19}$

Buchanan et al. support the idea that surgical complication avoidance is a key element to improving quality and preventing readmissions to the hospital. ${ }^{17}$ In their series of 5,569 patients with a $6.9 \%$ readmission rate, $50 \%$ were readmitted for a surgical complication, and only $22.9 \%$ for medical reasons. It is important to note that their series included adults and children, and thus a significant proportion of shunt-related problems. ${ }^{18}$ In our series, patients with shunt-related problems comprised $7.9 \%$ of the total readmitted population. The inclusion of pediatric patients results in a much higher representation of shunt complications, $21.1 \%$, in the readmitted population of the Buchanan paper. ${ }^{18}$. Surgical site infection was relatively uncommon, accounting for $8.0 \%$ of readmissions. Patients with a greater number of medical comorbidities in our study seem to be more likely to be readmitted. Indeed, they may have a greater risk of postoperative complications..$^{20,21}$ Other studies in the neurosurgical literature have found 
similar rates of readmission for medical reasons. ${ }^{11,13}$ Although reduction of surgical complications is a necessary goal and should be part of any effort to reduce readmission and improve quality, it is equally important to address the patients' medical comorbidities to reduce complications and thus readmissions.

Cox proportional hazard analysis was carried out to determine relationships between readmission and encounter type, gender, age, race, admission priority, discharge disposition, number of diagnoses, DRG-adjusted LOS, and insurance status. In this cohort, it was found that encounter type, gender, age, race, and elective vs emergency admission were not associated with an increased risk of readmission. Studies in the neurosurgical and orthopedic literature have found similarly that patients discharged to rehab facilities tend to be readmitted more often than patients discharged home $\mathrm{e}^{15,19,22}$, but this was not found to be the case in the present study. Of note was the finding that patients with emergency initial admissions were no more or less likely to be readmitted than those with elective admissions. The authors ascribe this to a number of factors - our institution, as a major referral center for trauma, services a significant trauma population, which tends to be more likely to be lost to follow up. ${ }^{23}$ These patients may be less likely to return if they are unwell. On the other hand, there may simply be little to no difference between the two groups with regard to who is more likely to return to the hospital.

In this study, average yearly readmission rate did show a strong negative linear correlation with hospital LOS. Our analysis indicates that for the whole population admitted to our institution between October 2007 and June 2014, the readmission rate rose as the LOS decreased. For individual patients, however, a longer length of stay was associated with a higher rate of readmission. These two conclusions seem to be at odds with each other, but an inspection of the remaining data can help elucidate an explanation. There is a significant association of comorbidity with both readmission and LOS (Tables $\underline{6 \text { and } 7}$, respectively). Thus patients who are in the hospital for a longer period of time are likely sicker than those who are admitted for a shorter period. These patients are also more likely to need readmission than their healthier counterparts. Unfortunately, a more detailed analysis to separate the effect of comorbidity on LOS and 
readmission was unable to be performed. This is a result of the large variability in the coding of comorbidities that were entered into the hospital administrative database.

Intuitively, this makes sense, because patients who come into the hospital with more comorbidities are less healthy than patients who come in with only the primary diagnosis for which they are being treated, and comorbidities make them more likely to have complications, both medical and surgical. A patient with poorly controlled diabetes, for example, would be more likely to develop problems with wound healing or infection. A patient with coronary artery or peripheral vascular disease whose aspirin is stopped due to intracranial hemorrhage may be more likely to develop ischemic events as a result. ${ }^{15}$ This association between comorbidity and readmission has been established in prior studies. ${ }^{21,24}$ Thus, readmission rate may be a poor surrogate marker for quality of care. Rather, it more accurately represents the level of acuity of the patient population at a given hospital. Furthermore, the real outcomes of interest should not be whether or not the patient is readmitted, but rather patients' quality of life and functional status after their hospitalization. Based on our study and others, it would seem that the focus should be shifted from hospital-centered metrics such as readmission rate to patient-centered metrics such as post-hospitalization quality of life. The effect of the healthcare delivery system on the patient's life is the real test of its quality.

\section{Limitations}

The primary limitation of this study is its reliance on a hospital administrative database. Many authors have commented on the inadequacy of these databases for providing a holistic picture of the patient's care. ${ }^{4,5,10,12,15,24-27}$. Indeed, hospital databases are highly dependent on the information entered into them, which depends on provider documentation and may not capture everything that is documented. Furthermore, the database's record keeping ends when the patient leaves the hospital, which eliminates the possibility of using the database to assess actual patient outcomes. Additionally, each hospital or system keeps its own database, which makes for inconsistency in information recorded and fragmentation of data. Also, readmissions to other hospitals would not be captured in our database. Documented reasons for readmission in our study were 
dependent on the diagnosis code entered and may not accurately reflect the real reason for patient readmission. These codes are often entered by coders who interpret provider notes, and this creates room for clerical error. As a result, this study has no ability to assess patient outcomes. This study is also retrospective, which limits its ability to draw conclusions regarding causation.

\section{CONCLUSION}

The analysis indicates that a decreasing LOS over time has been associated with an increasing readmission rate at the population level. However, at the individual level, a prolonged LOS is associated with a higher risk of readmission. The authors attribute this to patient comorbidities. However, this increasing readmission rate may represent a combination of factors including overall health and socioeconomic status of the patients being admitted. Thus the rate of readmission may represent a burden of illness rather than a valid metric for quality of care. 


\section{REFERENCES}

1. Jencks SF, Williams MV, Coleman EA. Rehospitalizations among patients in the Medicare fee-for-service program. The New England Journal of Medicine. 2009;360(14):1418-1428.

2. Axon RN, Williams MV. Hospital readmission as an accountability measure. Jama. 2011;305(5):504-505.

3. Commission MPA. Report to the Congress: Promoting Greater Efficiency in Medicare. Washington, DC, 2007.

4. Goff DC, Jr., Pandey DK, Chan FA, Ortiz C, Nichaman MZ. Congestive heart failure in the United States: is there more than meets the I(CD code)? The Corpus Christi Heart Project. Archives of Internal Medicine. 2000;160(2):197-202.

5. Ashton CM, Del Junco DJ, Souchek J, Wray NP, Mansyur CL. The association between the quality of inpatient care and early readmission: a meta-analysis of the evidence. Medical Care. 1997;35(10):1044-1059.

6. Ashton CM, Kuykendall DH, Johnson ML, Wray NP, Wu L. The association between the quality of inpatient care and early readmission. Annals of Internal Medicine. 1995;122(6):415-421.

7. Itani KMF. Longer length of stay or readmissions: can we achieve a balance? The American Journal of Surgery. 2014;207(4):457-458.

8. Keenan PS, Normand SL, Lin Z, et al. An administrative claims measure suitable for profiling hospital performance on the basis of 30-day all-cause readmission rates among patients with heart failure. Circulation. Cardiovascular Quality and Outcomes. 2008;1(1):29-37.

9. Physicians ACoE. Medicare's Hospital Readmission Reduction Program FAQ. Clinical and Practice Management 2015. Accessed 06/07, 2015.

10. Amin BY, Tu TH, Schairer WW, et al. Pitfalls of calculating hospital readmission rates based on nonvalidated administrative data sets: presented at the 2012 Joint Spine Section Meeting: clinical article. Journal of Neurosurgery. Spine. 2013;18(2):134-138.

11. Marcus LP, McCutcheon BA, Noorbakhsh A, et al. Incidence and predictors of 30-day readmission for patients discharged home after craniotomy for malignant 
supratentorial tumors in California (1995-2010). Journal of Neurosurgery. 2014;120(5):1201-1211.

12. McCormack RA, Hunter T, Ramos N, Michels R, Hutzler L, Bosco JA. An analysis of causes of readmission after spine surgery. Spine. 2012;37(14):12601266.

13. Shah MN, Stoev IT, Sanford DE, et al. Are readmission rates on a neurosurgical service indicators of quality of care? Journal of Neurosurgery. 2013;119(4):10431049.

14. Vaziri S, Cox JB, Friedman WA. Readmissions in Neurosurgery: A Qualitative Inquiry. World Neurosurgery. 2014.

15. Liotta EM, Singh M, Kosteva AR, et al. Predictors of 30-day readmission after intracerebral hemorrhage: a single-center approach for identifying potentially modifiable associations with readmission. Critical Care Medicine. 2013;41(12):2762-2769.

16. Singh M, Guth JC, Liotta E, et al. Predictors of 30-day readmission after subarachnoid hemorrhage. Neurocritical Care. 2013;19(3):306-310.

17. Vizient: Clinical Database. Irving, TX; 2016. https://cdprm.uhc.edu/CDPRM/web /reports/DefaultView.aspx. Accessed 25 October, 2016.

18. Buchanan CC, Hernandez EA, Anderson JM, et al. Analysis of 30-day readmissions among neurosurgical patients: surgical complication avoidance as key to quality improvement. Journal of Neurosurgery. 2014;121(1):170-175.

19. Lavernia CJ, Villa JM, Iacobelli DA. Readmission rates in the state of Florida: a reflection of quality? Clinical Orthopaedics and Related Research, 2013;471(12):3856-3862.

20. Avram V, Petruccelli D, Winemaker M, de Beer J. Total Joint Arthroplasty Readmission Rates and Reasons for 30-Day Hospital Readmission. The Journal of Arthroplasty. 2014;29(3):465-468.

21. Kohlnhofer BM, Tevis SE, Weber SM, Kennedy GD. Multiple complications and short length of stay are associated with postoperative readmissions. The American Journal of Surgery. 2014;207(4):449-456. 
22 Ramos NL, Karia RJ, Hutzler LH, Brandt AM, Slover JD, Bosco JA. The Effect of Discharge Disposition on 30-Day Readmission Rates After Total Joint Arthroplasty. The Journal of Arthroplasty. 2014;29(4):674-677.

23. Murnaghan ML, Buckley RE. Lost but not forgotten: patients lost to follow-up in a trauma database. Canadian Journal of Surgery. 2002;45(3):191-195.

24. Press MJ, Scanlon DP, Navathe AS, et al. The Importance of Clinical Severity in the Measurement of Hospital Readmission Rates for Medicare Beneficiaries, 1997-2007. Medical Care Research and Review. 2013;70(6):653-665.

25. Clement RC, Derman PB, Graham DS, Speck RM, Flynn DN, Levin LS, Fleisher LA. Risk Factors, Causes, and the Economic Implications of Unplanned Readmissions Following Total Hip Arthroplasty. The Journal of Arthroplasty. 2013;28(8, Suppl):7-10.

26. Hendren S, Morris AM, Zhang W, Dimick J. Early discharge and hospital readmission after colectomy for cancer. Diseases of the Colon and Rectum. 2011;54(11):1362-1367.

27 McCormack R, Michels R, Ramos N, Hutzler L, Slover JD, Bosco JA. Thirty-day readmission rates as a measure of quality: causes of readmission after orthopedic surgeries and accuracy of administrative data. Journal of Healthcare

Management. 2013;58(1):64-76; discussion 76-77. 


\section{FIGURE LEGENDS}

Figure 1. Flow diagram of patient selection process.

Figure 2. Plot of average yearly readmission rate with best-fit line.

Figure 3. Comparison of institutional data with comparable-size institutions. 
Table 1. Patient demographics

\begin{tabular}{|l|l|}
\hline Number of patient encounters & 9409 \\
\hline Number of readmissions & $925(9.8 \%)$ \\
\hline Number of males & $4686(49.8 \%)$ \\
\hline Elective admissions & $5916(62.8 \underline{9} \%)$ \\
\hline Average LOS & $6.19 \underline{21}$ days \\
\hline Average age & 55.2 years \\
\hline Number of patients expired & $330(3.4 \%)$ \\
\hline Abbreviations: LOS, length of hospital stay. & \\
\hline
\end{tabular}


Table 2. Classification of readmission

\begin{tabular}{|l|l|}
\hline Classification of Readmission & $N$ \\
\hline Elective & 240 \\
\hline Non-elective & 605 \\
\hline Undefined & 80 \\
\hline Total & 925 \\
\hline
\end{tabular}


Table 3. Patients with neurological reasons for readmission

\begin{tabular}{|l|l|}
\hline Reason for readmission & $\mathrm{N}(\%)$ \\
\hline $\begin{array}{l}\text { Postoperative hemorrhage or CSF leak requiring } \\
\text { surgery }\end{array}$ & $184(54.4)$ \\
\hline Meningitis & $24(7.1)$ \\
\hline Postoperative pain & $39(11.5)$ \\
\hline Seizure & $23(6.8)$ \\
\hline Hydrocephalus & $16(4.7)$ \\
\hline Encephalopathy & $12(3.5)$ \\
\hline Stroke/Ischemia & $40(11.8)$ \\
\hline Abbreviations: CSF, cerebrospinal fluid. & \\
\hline
\end{tabular}


Table 4. Breakdown of medical comorbidities resulting in readmission

\begin{tabular}{|l|l|}
\hline System/Comorbidity & $\mathrm{N}(\%)$ \\
\hline Gastrointestinal & $68(18.8)$ \\
\hline Cardiac & $49(13.5)$ \\
\hline Renal/Urinary (including UTI) & $49(13.5)$ \\
\hline Endocrine (including diabetes) & $15(4.1)$ \\
\hline Pulmonary & $38(10.5)$ \\
\hline Hematologic/VTE & $63(17.4)$ \\
\hline Oncologic & $50(13.8)$ \\
\hline Drug related & $30(8.3)$ \\
\hline Abbreviations: UTI, urinary tract infection; VTE, venous thromboembolism. \\
\hline
\end{tabular}


Table 5. Insurance status and readmission

\begin{tabular}{|l|l|l|}
\hline Insurance types & N & Readmits \\
\hline Commercial & 3959 & 322 \\
\hline Medicaid & 806 & 91 \\
\hline Medicare & 3805 & 433 \\
\hline Self pay & 533 & 56 \\
\hline Other government & 235 & 20 \\
\hline Workman's comp & 71 & 3 \\
\hline
\end{tabular}


Table 6. Number of diagnoses and readmission

\begin{tabular}{|l|l|}
\hline Diagnoses & Readmitted (\%) \\
\hline Less than 10 & $413(8.53)$ \\
\hline More than 10 & $512(11.38)$ \\
\hline & $\mathrm{p}<0.001$ \\
\hline
\end{tabular}


Table 7. LOS as a function of number of diagnoses

\begin{tabular}{|l|l|}
\hline & LOS $( \pm S D)$ \\
\hline$<10$ diagnoses & $4.00( \pm 3.08)$ \\
\hline$>10$ diagnoses & $9.00( \pm 9.04)$ \\
\hline $\mathrm{p}<0.01$ & \\
\hline $\begin{array}{l}\text { Abbreviations: } \text { LOS, length of hospital stay; } \\
\text { SD, standard deviation. }\end{array}$ \\
\hline
\end{tabular}


Table 8. Result of multivariate analysis

\begin{tabular}{|c|c|c|c|}
\hline Parameter & Group & Hazard Ratio $(95 \% \mathrm{Cl})$ & $\mathrm{p}$ \\
\hline \multirow[t]{4}{*}{ Adjusted LOS } & 1 (0-25\%tile) & 1 & 0.25 \\
\hline & 2 (26-50\%tile) & $1.188(0.888,1.591)$ & 0.15 \\
\hline & 3 (51-75\%tile) & $1.249(0.924,1.688)$ & 0.15 \\
\hline & 4 (76-100\%tile) & $1.425(1.032,1.967)$ & 0.03 \\
\hline \multirow[t]{2}{*}{ Gender } & Female & 1 & \\
\hline & Male & $1.045(0.892,1.224)$ & 0.58 \\
\hline \multirow[t]{2}{*}{ Age } & $<65$ & 1 & \\
\hline & $\geq 65$ & $0.844(0.697,1.023)$ & 0.08 \\
\hline \multirow[t]{2}{*}{ Race } & White & 1 & \\
\hline & Other & $1.067(0.849,1.341)$ & 0.58 \\
\hline \multirow[t]{2}{*}{ Admission Priority } & Emergency & 1 & \\
\hline & Elective & $0.983(0.812,1.185)$ & 0.86 \\
\hline \multirow[t]{2}{*}{ Discharge Disposition } & Home & ( & \\
\hline & Rehab/SNF & $1.03(0.845,1.255)$ & 0.77 \\
\hline \multirow[t]{2}{*}{ Number of Diagnoses } & $<10$ & 1 & \\
\hline & $>10$ & $1.912(0.99,1.436)$ & 0.06 \\
\hline \multirow[t]{2}{*}{ MS_DRG } & Surgical & 1 & \\
\hline & Medical & $1.219(0.904,1.646)$ & 0.19 \\
\hline \multirow[t]{4}{*}{ Insurance } & Commercial & 1 & \\
\hline & Medicare & $1.425(1.167,1.741)$ & $<0.01$ \\
\hline & Medicaid & $1.328(0.998,1.767)$ & 0.05 \\
\hline & Other & $1.035(0.759,1.410)$ & 0.83 \\
\hline
\end{tabular}


All neurosurgical patient encounters

$$
\text { ( } n=9739 \text { ) }
$$

Deceased ( $n=330)$
Elective admissions

$$
(n=5916 ; 62.9 \%)
$$

Urgent/emergent admissions

$(n=3493 ; 37.2 \%)$ 


\section{Readmission v.s. LOS}

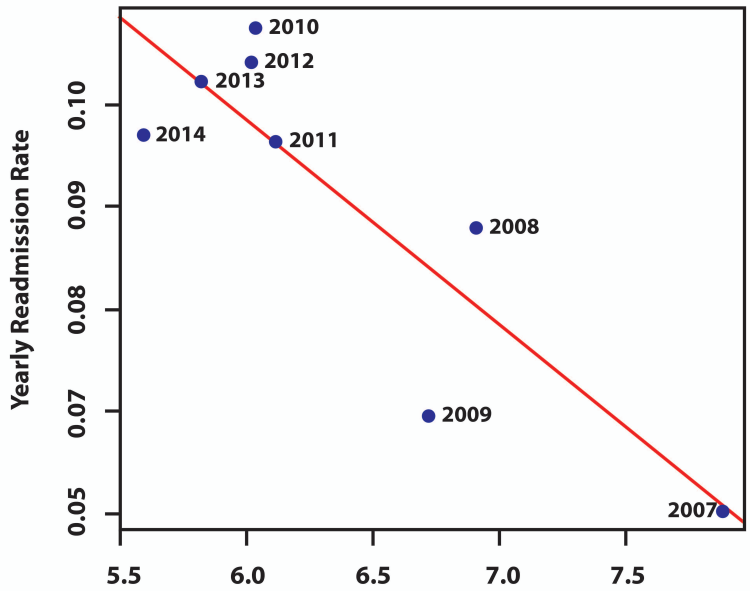




\section{Institution Comparison, LOS and Readmission}



